\title{
Irreversibility inversions in two-dimensional turbulence
}

\author{
Andrew D. Bragg* \\ Department of Civil and Environmental Engineering, Duke University, Durham, North Carolina 27708, USA \\ Filippo De Lillo and Guido Boffetta \\ Department of Physics and INFN, University of Torino, Via Pietro Giuria 1, 10125 Torino, Italy
}

(Received 26 September 2017; published 12 February 2018)

\begin{abstract}
In this paper, we consider a recent theoretical prediction [Bragg et al., Phys. Fluids 28, 013305 (2016)] that for inertial particles in two-dimensional (2D) turbulence, the nature of the irreversibility of the particle-pair dispersion inverts when the particle inertia exceeds a certain value. In particular, when the particle Stokes number, $\mathrm{St}$, is below a certain value, the forward-in-time (FIT) dispersion should be faster than the backward-in-time (BIT) dispersion, but for St above this value, this should invert so that BIT becomes faster than FIT dispersion. This nontrivial behavior arises because of the competition between two physically distinct irreversibility mechanisms that operate in different regimes of St. In three-dimensional (3D) turbulence, both mechanisms act to produce faster BIT than FIT dispersion, but in 2D turbulence, the two mechanisms have opposite effects because of the flux of energy from the small to the large scales. We supplement the qualitative argument given by Bragg et al. [Phys. Fluids 28, 013305 (2016)] by deriving quantitative predictions of this effect in the short time limit. We confirm the theoretical predictions using results of inertial particle dispersion in a direct numerical simulation of $2 \mathrm{D}$ turbulence. A more general finding of this analysis is that in turbulent flows with an inverse energy flux, inertial particles may yet exhibit a net downscale flux of kinetic energy because of their nonlocal-in-time dynamics.
\end{abstract}

DOI: 10.1103/PhysRevFluids.3.024302

\section{INTRODUCTION}

The problem of particle dispersion in turbulence is important from both fundamental and practical perspectives. From a fundamental perspective, it is important because such studies relate to the Lagrangian dynamics of turbulent flows, whose study has proven to reveal deep and interesting things about turbulence [1]. From a practical perspective, the subject is important because of its implications for problems such as pollutant dispersion, droplet mixing in clouds, and the distribution of plankton in oceans, to name but a few.

An important problem concerns the irreversibility of multiparticle dispersion in turbulence. For the case of fluid particles, irreversibility in their multiparticle dispersion is expected due to the net flux of kinetic energy among the scales of motion of the turbulence, which is the signature of irreversibility in the underlying Eulerian velocity field. Irreversibility is important not only from a theoretical perspective but also because irreversible dispersion means that particles spread out and mix together in turbulence at different rates [2], with important implications for modeling such problems. Understanding various manifestations of Lagrangian irreversibility and its relation to irreversibility in

*andrew.bragg@duke.edu 
the underlying Eulerian turbulent velocity field is something that has recently attracted considerable attention [2-11].

In many real systems, the particles suspended in the turbulent flow are not fluid particles (tracers), but often have inertia and are polydisperse and nonspherical, along with many other complexities. These features can cause the particle motion to differ in striking ways from that of fluid particles [12], and so understanding the effect of these complexities on the way the particles disperse in turbulence is an important problem. In a recent paper, Bragg et al. [2] investigated theoretically and computationally how inertia affects particle dispersion and its irreversibility in turbulence. They showed that in three-dimensional (3D) turbulence, inertia can affect the dispersion in very profound ways and can have a strong effect upon the irreversibility. They argued that whereas the irreversibility of the fluid particle dispersion arises due to fluxes in the underlying turbulent velocity field, inertial particles experience an additional mechanism owing to their nonlocal-in-time dynamics. It was shown that this additional mechanism generates inertial particle dispersion that can be much more strongly irreversible than that of fluid particles, with the backward-in-time (BIT) mean-square separation of the particles being up to an order of magnitude faster than the forward-in-time (FIT) counterpart in some cases.

Because both mechanisms lead to a faster BIT dispersion, it is not immediately clear how to distinguish between the two effects in realistic flows, without relying on synthetic velocity fields with ad hoc statistical properties. However, a suggestion comes from the result that the asymmetry in the fluid particle dispersion, for two particles initially at separation $r$, ultimately depends on the sign of the upscale energy flux through scale $r, \mathcal{F}(r)$. Indeed, the faster BIT separation observed in $3 \mathrm{D}$ turbulence is due to the fact that, according to the Kolmogorov's four-fifths law, in the inertial range $\mathcal{F}(r)=-\langle\epsilon\rangle<0$, where $\langle\epsilon\rangle$ is the kinetic energy dissipation rate. If one instead considers two-dimensional (2D) turbulence, the same law can be generalized to show that $\mathcal{F}(r)>0$, leading to faster separation FIT than BIT, thus reversing the asymmetry. This has in fact already been observed for fluid particles in Ref. [13]. On the other hand, the irreversibility mechanism intrinsic in the dynamics of heavy particles does not depend on the details of the fluid flow and will still favor BIT separation. While the latter should dominate for particles with a large inertia, thus giving faster BIT separation, tracers and weakly inertial particles should separate faster FIT. A transition between the two behaviors should be observed in the inertia parameter, the Stokes number St. In the present paper, we supplement this qualitative argument for the irreversibility inversions with a quantitative analysis in the short time regime.

We test the predictions for the irreversibility inversions using direct numerical simulations (DNS) of inertial particle dispersion in $2 \mathrm{D}$ turbulence. The presence of a transition between the two behaviors would signal the presence of two entirely distinct physical mechanisms generating the irreversibility of inertial particle dispersion in turbulence. In recent years, not only has $2 \mathrm{D}$ turbulence been studied in depth (see Ref. [14] and references therein) but also the dynamics of inertial particles in 2D turbulence, e.g., Refs. [13,15-19].

Further, 2D turbulence describes behavior that is not destroyed by perturbations in the third dimension of quasi-2D (Q2D) turbulent flows. Such Q2D flows can occur in nature either because of geometrical constraints on the flow or because of imposed body forces [14]. In particular, in Q2D turbulent flows, one can observe $\mathcal{F}(r)>0$ over a range of $r$ [20]. As such, understanding inertial particle motion and the irreversibility of their dispersion in 2D turbulence can have applications to Q2D turbulent flows that occur in geophysical and astrophysical contexts [21].

The outline of the rest of the paper is as follows. In Sec. II, we review the physical mechanisms for irreversible inertial particle dispersion in turbulence and explain the interesting qualitative prediction that they give rise to for 2D turbulence. In Sec. II, we also derive a new quantitative result for dispersion in the short-time regime that supports the qualitative predictions. Then, in Sec. III, we use data from direct numerical simulations (DNS) to test the prediction and the underlying explanations. Section IV is devoted to conclusions. 


\section{THEORY}

We consider monodisperse inertial particle pairs subject to Stokes drag forcing only [22], whose equation of relative motion is

$$
\ddot{\boldsymbol{r}}^{p}(t) \equiv \dot{\boldsymbol{w}}^{p}(t)=\frac{1}{\tau_{p}}\left[\Delta \boldsymbol{u}\left(\boldsymbol{x}^{p}(t), \boldsymbol{r}^{p}(t), t\right)-\boldsymbol{w}^{p}(t)\right],
$$

where $\boldsymbol{x}^{p}(t)$ and $\boldsymbol{x}^{p}(t)+\boldsymbol{r}^{p}(t)$ are the positions of the two particles, $\boldsymbol{w}^{p}(t)$ their relative velocity, and $\Delta \boldsymbol{u}\left(\boldsymbol{x}^{p}(t), \boldsymbol{r}^{p}(t), t\right)$ is the difference in the fluid velocity evaluated at the particle positions [2]. In this paper, we shall be interested in the case where the system is turbulent, with statistics that are stationary, homogeneous, and isotropic. As a consequence of the homogeneity, when $\Delta \boldsymbol{u}\left(\boldsymbol{x}^{p}(t), \boldsymbol{r}^{p}(t), t\right)$ appears in statistical expressions, we shall drop the $\boldsymbol{x}^{p}(t)$ argument.

In the limit $\tau_{p} \rightarrow 0$, the particles represent fluid particles whose equation of relative motion is

$$
\dot{\boldsymbol{r}}^{f}(t) \equiv \Delta \boldsymbol{u}\left(\boldsymbol{x}^{f}(t), \boldsymbol{r}^{f}(t), t\right)
$$

where the superscript $p$ has been replaced with $f$ to denote that these are fluid particles. The FIT and BIT mean-square separations of the particles are denoted by $\left\langle\left\|\boldsymbol{r}^{p}(t)\right\|^{2}\right\rangle_{\xi},\left\langle\left\|\boldsymbol{r}^{p}(-t)\right\|^{2}\right\rangle_{\xi}$, where $\langle\cdot\rangle_{\xi}$ denotes an ensemble average conditioned on $\boldsymbol{r}^{p}(0)=\xi$. The conditioning time can be set to zero since we are interested in statistically stationary flows.

In Bragg et al. [2], it was argued that for inertial particles, there are two distinct mechanisms that generate irreversible dispersion, i.e., $\left\langle\left\|\boldsymbol{r}^{p}(t)\right\|^{2}\right\rangle_{\xi} \neq\left\langle\left\|\boldsymbol{r}^{p}(-t)\right\|^{2}\right\rangle_{\xi}$. Here we summarize the conceptual ideas and refer the readers to that paper for detailed arguments. First, we define a scale-dependent Stokes number $\operatorname{St}_{r}(t) \equiv \tau_{p} / \tau_{r}(t)$, where $\tau_{r}(t)$ is the eddy turnover time evaluated at the scale $\left\|\boldsymbol{r}^{p}(t)\right\|$. Next, we note that the quantities $\left\langle\left\|\boldsymbol{r}^{p}(t)\right\|^{2}\right\rangle_{\xi}$ and $\left\langle\left\|\boldsymbol{r}^{p}(-t)\right\|^{2}\right\rangle_{\xi}$ are dominated by the behavior of particle pairs that move apart and particle pairs that move together, respectively.

When $\mathrm{St}_{\xi} \ll 1$, the effect of inertia is weak, and the dispersion irreversibility arises because the energy flux in the turbulent field $\Delta \boldsymbol{u}$ causes particle pairs to move together and apart at characteristically different rates. In 3D turbulence $\mathcal{F}(r)<0$, and this causes the particle pairs to move together more energetically than apart, leading to $\left.\left\langle\left\|\boldsymbol{r}^{p}(-t)\right\|^{2}\right\rangle_{\xi}\right\rangle\left\langle\left\|\boldsymbol{r}^{p}(t)\right\|^{2}\right\rangle_{\xi}$. However, in 2D turbulence $\mathcal{F}(r)<0$, thus leading to the opposite behavior $\left\langle\left\|\boldsymbol{r}^{p}(t)\right\|^{2}\right\rangle_{\xi}>\left\langle\left\|\boldsymbol{r}^{p}(-t)\right\|^{2}\right\rangle_{\xi}$. This is referred to as the local irreversibility mechanism (LIM) [2], since it arises from the behavior of the local turbulence experienced by the particles.

When $\mathrm{St}_{\xi} \geqslant O(1)$, the inertial particle relative motion is strongly affected by their nonlocal-intime dynamics, which gives rise to a "path-history effect" when the statistics of $\Delta \boldsymbol{u}$ depend upon separation [23]. This effect arises since particle-pair motion depends upon the multiscale nature of turbulence, and as inertial particles posses memory, their motion can be influenced by their interaction with turbulent scales in the past that had properties very different from the scales associated with their current separation. In particular, particle pairs moving together will carry with them a memory of their interaction with scales larger than those at their current separation, whereas particle pairs moving apart will carry with them a memory of their interaction with scales smaller than those at their current separation. This path history effect leads to downscale energy fluxes in the inertial particle-pair motion [24,25], associated with inertial particles moving together more energetically than apart, and hence leads to $\left.\left\langle\left\|\boldsymbol{r}^{p}(-t)\right\|^{2}\right\rangle_{\xi}\right\rangle\left\langle\left\|\boldsymbol{r}^{p}(t)\right\|^{2}\right\rangle_{\xi}$. In Bragg et al. [2], this was refereed to as the nonlocal irreversibility mechanism (NLIM).

That the NLIM generates faster BIT than FIT dispersion only depends upon the fluid having the property that the statistics of $\Delta \boldsymbol{u}$ increase with increasing separation. Crucially, unlike the LIM, the NLIM does not depend upon the sign of $\mathcal{F}$. This then leads to an interesting prediction: In 2D turbulence, $\left\langle\left\|\boldsymbol{r}^{p}(t)\right\|^{2}\right\rangle_{\xi}>\left\langle\left\|\boldsymbol{r}^{p}(-t)\right\|^{2}\right\rangle_{\xi}$ until $\mathrm{St}_{\xi}$ becomes large enough and then $\left.\left\langle\left\|\boldsymbol{r}^{p}(-t)\right\|^{2}\right\rangle_{\xi}\right\rangle$ $\left\langle\left\|\boldsymbol{r}^{p}(t)\right\|^{2}\right\rangle_{\xi}$, i.e., an inversion in the nature of the dispersion irreversibility as the particle inertia is increased. 


\section{A. Short-time analysis}

The preceding qualitative argument for the irreversibility inversion may be supplemented by a quantitative analysis of the dispersion in the short-time limit. Using an expansion in $t$, we may write $[4,8]$

$$
\left\langle\left\|\boldsymbol{r}^{f}(t)\right\|^{2}-\left\|\boldsymbol{r}^{f}(-t)\right\|^{2}\right\rangle_{\xi}=2\langle\Delta \boldsymbol{u}(\boldsymbol{\xi}, 0) \cdot \Delta \boldsymbol{a}(\boldsymbol{\xi}, 0)\rangle t^{3}+\mathcal{O}\left(t^{5}\right),
$$

where $\Delta \boldsymbol{a}(\boldsymbol{\xi}, 0)$ is the difference in the fluid acceleration field $\boldsymbol{a} \equiv \partial_{t} \boldsymbol{u}+(\boldsymbol{u} \cdot \nabla) \boldsymbol{u}$ evaluated at two points separated by $\xi$. Using results from Lindborg [26], we can derive the following result for statistically stationary, isotropic, 2D turbulence:

$$
\begin{aligned}
\langle\Delta \boldsymbol{u}(\boldsymbol{\xi}, 0) \cdot \Delta \boldsymbol{a}(\boldsymbol{\xi}, 0)\rangle & =\frac{1}{2} \nabla_{\xi} \cdot\left\langle\Delta \boldsymbol{u}(\boldsymbol{\xi}, 0)\|\Delta \boldsymbol{u}(\boldsymbol{\xi}, 0)\|^{2}\right\rangle \\
& =\frac{1}{6}\left(\xi \nabla_{\xi}^{2} S_{3, \|}^{f}+8 \nabla_{\xi} S_{3, \|}^{f}\right),
\end{aligned}
$$

where $S_{3, \|}^{f}(\xi) \equiv\left\langle\left[\Delta u_{\|}(\xi, 0)\right]^{3}\right\rangle$ and $\xi \equiv\|\xi\|$. For forced 2D turbulence, with forcing lengthscale $\ell_{f}$, we have the double cascade scenario for which [14]

$$
\begin{aligned}
& S_{3, \|}^{f}(\xi)=\frac{1}{8} \Omega \xi^{3}, \text { for } \xi \ll \ell_{f}, \\
& S_{3, \|}^{f}(\xi)=\frac{3}{2} \varepsilon \xi, \quad \text { for } \xi \gg \ell_{f},
\end{aligned}
$$

where $\Omega$ is the enstrophy dissipation by viscosity and $\varepsilon$ is the upscale energy flux. From (5) and (6), we see that the contribution in (3) is always positive but with different $\xi$ dependence for the direct and the inverse cascade regimes. Substituting (5) and (6) into (4) gives

$$
\begin{gathered}
\left\langle\left\|\boldsymbol{r}^{f}(t)\right\|^{2}-\left\|\boldsymbol{r}^{f}(-t)\right\|^{2}\right\rangle_{\xi}=\frac{5}{8} \Omega \xi^{2} t^{3}+\mathcal{O}\left(t^{5}\right), \quad \text { for } \xi \ll \ell_{f}, \\
\left\langle\left\|\boldsymbol{r}^{f}(t)\right\|^{2}-\left\|\boldsymbol{r}^{f}(-t)\right\|^{2}\right\rangle_{\xi}=2 \varepsilon t^{3}+O\left(t^{5}\right), \quad \text { for } \xi \gg \ell_{f},
\end{gathered}
$$

and these are positive, showing that FIT dispersion is faster than BIT dispersion in both the direct and inverse cascade regimes of $2 \mathrm{D}$ turbulence.

For inertial particles, the result corresponding to (3) is

$$
\left\langle\left\|\boldsymbol{r}^{p}(t)\right\|^{2}-\left\|\boldsymbol{r}^{p}(-t)\right\|^{2}\right\rangle_{\xi}=2\left\langle\boldsymbol{w}^{p}(0) \cdot \dot{\boldsymbol{w}}^{p}(0)\right\rangle_{\xi} t^{3}+\mathcal{O}\left(t^{5}\right) .
$$

To express $\left\langle\boldsymbol{w}^{p}(0) \cdot \dot{\boldsymbol{w}}^{p}(0)\right\rangle_{\xi}$ in the form of a flux, analogous to (4), we use the evolution equation for the PDF $p(\boldsymbol{\xi}, \boldsymbol{w}, t) \equiv\left\langle\delta\left(\boldsymbol{r}^{p}(t)-\boldsymbol{\xi}\right) \delta\left(\boldsymbol{w}^{p}(t)-\boldsymbol{w}\right)\right\rangle$, namely [27]

$$
\partial_{t} p=-\nabla_{\xi} \cdot p \boldsymbol{w}-\nabla_{\boldsymbol{w}} \cdot p\left\langle\dot{\boldsymbol{w}}^{p}(t)\right\rangle_{\xi, \boldsymbol{w}} .
$$

Multiplying the stationary form of (10) by $\|\boldsymbol{w}\|^{2}$ and then integrating over all $\boldsymbol{w}$ allows us to derive the following result for a statistically stationary, isotropic system,

$$
\left\langle\boldsymbol{w}^{p}(t) \cdot \dot{\boldsymbol{w}}^{p}(t)\right\rangle_{\xi}=\frac{1}{2 g} \nabla_{\xi} \cdot g\left\langle\boldsymbol{w}^{p}(t)\left\|\boldsymbol{w}^{p}(t)\right\|^{2}\right\rangle_{\xi}
$$

where [24]

$$
g(\xi) \equiv \frac{N(N-1)}{n^{2} V} \int_{\mathbb{R}^{3}} p d \boldsymbol{w}
$$

is the radial distribution function (RDF), $N$ is the total number of particles in the control volume $V$, and $n \equiv N / V$.

Using the equation of motion for $\boldsymbol{w}^{p}$, we may write

$$
\left\langle\boldsymbol{w}^{p}(0) \cdot \dot{\boldsymbol{w}}^{p}(0)\right\rangle_{\xi}=\left\langle\Delta \boldsymbol{u}\left(\boldsymbol{r}^{p}(0), 0\right) \cdot \boldsymbol{w}^{p}(0)\right\rangle_{\xi}-\left\langle\left\|\boldsymbol{w}^{p}(0)\right\|^{2}\right\rangle_{\xi}
$$


In the regime $\mathrm{St}_{\xi}(t) \ll 1, \boldsymbol{w}^{p}(t)=\Delta \boldsymbol{u}\left(\boldsymbol{r}^{p}(t), t\right)-\mathrm{St}_{\xi} \tau_{\xi} \Delta \boldsymbol{a}\left(\boldsymbol{r}^{p}(t), t\right)+O\left(\mathrm{St}_{\xi}^{2}\right), \quad$ where $\Delta \boldsymbol{a}\left(\boldsymbol{r}^{p}(t), t\right)$ is the difference in the fluid acceleration field evaluated at the positions of the two inertial particles. In this case, we find for $\mathrm{St}_{\xi} \ll 1$

$$
\left\langle\boldsymbol{w}^{p}(0) \cdot \dot{\boldsymbol{w}}^{p}(0)\right\rangle_{\xi}=\left\langle\Delta \boldsymbol{u}\left(\boldsymbol{r}^{p}(0), 0\right) \cdot \Delta \boldsymbol{a}\left(\boldsymbol{r}^{p}(0), 0\right)\right\rangle_{\xi}-\mathrm{St}_{\xi} \tau_{\xi}\left\langle\left\|\Delta \boldsymbol{a}\left(\boldsymbol{r}^{p}(0), 0\right)\right\|^{2}\right\rangle_{\xi}
$$

The result in (14) then implies through (9) that in the regime $\mathrm{St}_{\xi} \ll 1$, inertial particles will separate faster FIT than BIT in 2D turbulence, just as is the case for tracers. Note that this involves the assumption that $\left\langle\Delta \boldsymbol{u}\left(\boldsymbol{r}^{p}(0), 0\right) \cdot \Delta \boldsymbol{a}\left(\boldsymbol{r}^{p}(0), 0\right)\right\rangle_{\boldsymbol{\xi}}$ differs from $\langle\Delta \boldsymbol{u}(\boldsymbol{\xi}, 0) \cdot \Delta \boldsymbol{a}(\boldsymbol{\xi}, 0)\rangle$ in magnitude, but not in sign. This seems very reasonable, especially since preferential sampling is not too strong for $\mathrm{St}_{\xi} \ll 1$. The term of order $\mathrm{St}_{\xi}$ indicates that in this regime inertia acts to reduce the asymmetry.

In the regime $\mathrm{St}_{\xi} \geqslant O(1)$, something very different can occur. In particular, (13) shows that if $\left\langle\left\|\boldsymbol{w}^{p}(0)\right\|^{2}\right\rangle_{\boldsymbol{\xi}}>\left\langle\Delta \boldsymbol{u}\left(\boldsymbol{r}^{p}(0), 0\right) \cdot \boldsymbol{w}^{p}(0)\right\rangle_{\xi}$ then we can have $\left\langle\boldsymbol{w}^{p}(0) \cdot \dot{\boldsymbol{w}}^{p}(0)\right\rangle_{\xi}<0$, i.e., that although tracer particles separate faster FIT than BIT in 2D turbulence, inertial particles with $\mathrm{St}_{\xi} \geqslant O(1)$ can separate faster BIT than FIT, an inversion in the nature of the two-particle dispersion irreversibility. This also means through (11), that the inertial particle pairs would experience a downscale flux of kinetic energy, opposite in sign to the fluid energy flux $\mathcal{F}(r)>0$ for 2D turbulence.

The behavior $\left.\left\langle\left\|\boldsymbol{w}^{p}(0)\right\|^{2}\right\rangle_{\xi}\right\rangle\left\langle\Delta \boldsymbol{u}\left(\boldsymbol{r}^{p}(0), 0\right) \cdot \boldsymbol{w}^{p}(0)\right\rangle_{\xi}$ can arise in the regime $\mathrm{St}_{\xi} \geqslant O(1)$, at subintegral scales (i.e., where the statistics of $\Delta \boldsymbol{u}$ depend upon separation), through the "pathhistory effect" [24,28] described earlier. Recall that this effect describes the fact that since inertial particles possess memory, they can remember their interaction with turbulent scales along their path history that were larger and more energetic than those at their current separation, giving rise to $\left\|\boldsymbol{w}^{p}(t)\right\|>\left\|\Delta \boldsymbol{u}\left(\boldsymbol{r}^{p}(t), t\right)\right\|$, in a statistical sense. Although the effect operates at all subintegral scales in turbulence, it is most effective in the dissipation range (i.e., where the velocity field is smooth) where it gives rise to "caustics" [29], characterized by $\left\|\boldsymbol{w}^{p}\right\| \gg\left\|\Delta \boldsymbol{u}\left(\boldsymbol{r}^{p}(t), t\right)\right\|$.

Another important mechanism influencing $\boldsymbol{w}^{p}(t)$ in turbulence is the preferential sampling effect, wherein because of their inertia, inertial particles tend to avoid vorticity-dominated regions of the flow [30]. At inertial range scales, preferential sampling is associated with the inertial particles avoiding regions where the coarse-grained vorticity dominates over the coarse-grained straining motions of the turbulence [27]. Although the energy flux $\left(\tau_{p} / 2 g\right) \nabla_{\xi} \cdot g\left\langle\boldsymbol{w}^{p}(t)\left\|\boldsymbol{w}^{p}(t)\right\|^{2}\right\rangle_{\xi}$ in (11) is certainly affected by preferential sampling, it is the path-history effect, and not preferential sampling, that should be understood as the fundamental cause of the flux inversions described above. One argument for this is the fact that the above discussion also applies to flows in which the temporal evolution of $\Delta \boldsymbol{u}\left(\boldsymbol{r}^{p}(t), t\right)$ is white in time, since the path-history effect still operates in such a flow [31]. However, in white-in-time flows, the preferential sampling effect is absent [32].

In summary then, our arguments predict that although particle pairs separate faster FIT than BIT when $\mathrm{St}_{\xi} \ll 1$, as $\mathrm{St}_{\xi}$ is increased, this behavior can invert, causing particle pairs to separate faster BIT than FIT. This inversion occurs because when $\mathrm{St}_{\xi}$ is small, the direction of the particle-pair energy flux is governed by the flux in the turbulent velocity field, which is upscale in 2D turbulence. However, as $\mathrm{St}_{\xi}$ is increased, the nonlocal or path-history contribution to their dynamics becomes important, and this always causes the flux to be downscale.

Unlike the fluid particle case, we are not able in general to derive an analytical prediction for $\left\langle\left\|\boldsymbol{r}^{p}(t)\right\|^{2}-\left\|\boldsymbol{r}^{p}(-t)\right\|^{2}\right\rangle_{\xi}$ in the short-time regime since analytical results for $\left(\tau_{p} / 2 g\right) \nabla_{\xi} \cdot g\left\langle\boldsymbol{w}^{p}(t)\left\|\boldsymbol{w}^{p}(t)\right\|^{2}\right\rangle_{\xi}$ are not in general available for $\mathrm{St}_{\xi} \geqslant O(1)$. However, analytical results for the statistics of $\boldsymbol{w}^{p}(t)$ in the limit $\xi \rightarrow 0$ and for $\mathrm{St}_{\xi} \geqslant O(1)$ have been derived by Gustavsson and Mehlig [33] that apply to 2D flows. Here, we make use of those results to derive a prediction for the short-time behavior of $\left\langle\left\|\boldsymbol{r}^{p}(t)\right\|^{2}-\left\|\boldsymbol{r}^{p}(-t)\right\|^{2}\right\rangle_{\xi}$ when $\mathrm{St}_{\xi} \geqslant O(1)$ and $\xi \ll \ell_{f}$.

Substituting (11) into (9), and invoking the isotropy of the system, we obtain

$$
\left\langle\left\|\boldsymbol{r}^{p}(t)\right\|^{2}-\left\|\boldsymbol{r}^{p}(-t)\right\|^{2}\right\rangle_{\xi}=\left(\left[\nabla_{\xi} S_{3, \|}^{p}+\nabla_{\xi} S_{3, \|, \perp}^{p}+4 \xi^{-1} S_{3, \| \perp}^{p}\right]+\left[S_{3, \|}^{p}+S_{3, \|, \perp}^{p}\right] \nabla_{\xi} \ln g\right) t^{3}
$$


where $S_{3, \|}^{p}(\xi, t) \equiv\left\langle\left[w_{\|}^{p}(t)\right]^{3}\right\rangle_{\xi}$ and $S_{3, \| \perp}^{p}(\xi, t) \equiv\left\langle w_{\|}^{p}(t)\left[w_{\perp}^{p}(t)\right]^{2}\right\rangle_{\xi}$. We will now demonstrate that when $\tau_{p}$ is large enough for the nonlocal dynamics to control the inertial particle relative velocities at $\xi \ll \ell_{f}$, the sign of (15) is negative, in contrast to (7), which is positive.

When the nonlocal dynamics control the inertial particle velocities at small separations, "caustics" form [33], in which the statistics of $w_{\|}^{p}(t)$ and $w_{\perp}^{p}(t)$ become approximately equal [28], so that $S_{3, \|}^{p} \approx S_{3, \| \perp}^{p}$. In the caustic regime, the structure functions $S_{N, \|}^{p}(\xi, t) \equiv\left\langle\left[w_{\|}^{p}(t)\right]^{N}\right\rangle_{\xi}$ exhibit power-law behavior [33] such that $S_{3, \|}^{p}=\alpha_{3} \xi^{\beta_{3}}, g(\xi)=\alpha_{0} \xi^{-\beta_{0}}$, where $\alpha_{0}, \beta_{3}, \beta_{0}$ are all positive (we shall return to $\alpha_{3}$ shortly). Substituting these results into (15) gives

$$
\left\langle\left\|\boldsymbol{r}^{p}(t)\right\|^{2}-\left\|\boldsymbol{r}^{p}(-t)\right\|^{2}\right\rangle_{\xi}=2 \alpha_{3}\left(2+\beta_{3}-\beta_{0}\right) \xi^{\beta_{3}-1} t^{3} .
$$

The exponents take on values $\beta_{3} \in[0,3]$, where $\beta_{3}=0$ corresponds to the ballistic limit, and $\beta_{0} \in[0,1)$ in $2 \mathrm{D}$ turbulence (e.g., Boffetta et al. [15]). Consequently, $2+\beta_{3}-\beta_{0}>0$, and so the sign of (16) is determined by the sign of $\alpha_{3}$ which corresponds to the sign of $S_{3, \|}^{p}$. This is precisely what is expected given the irreversibility mechanisms explained earlier, and those arguments predict that when the nonlocal dynamics of the inertial particles dominate their motion then $\alpha_{3}<0$, corresponding to the particle pairs approaching more energetically than they separate.

Taken together, the results in (7) and (16) predict that in the direct cascade regime of 2D turbulence, $\left\langle\left\|\boldsymbol{r}^{p}(t)\right\|^{2}\right\rangle_{\xi}-\left\langle\left\|\boldsymbol{r}^{p}(-t)\right\|^{2}\right\rangle_{\xi}$ will be positive for $\mathrm{St}_{\xi}=0$, but will invert and become negative once $\mathrm{St}_{\xi}$ is large enough for the path-history effect to dominate the particle relative velocities.

\section{RESULTS AND DISCUSSION}

In order to test the predictions from Sec. II, we perform extensive direct numerical simulations (DNS) of particle-pair dispersion in 2D turbulence. Owing to the very high resolutions that are required in order to accurately resolve both the direct and inverse cascades of 2D turbulence, we here focus on the dispersion in the inverse cascade regime and will consider the behavior in the direct cascade regime in a future work.

We integrate the Navier-Stokes equation for the vorticity field $\omega \equiv \nabla \times \boldsymbol{u}$

$$
\partial_{t} \omega+\boldsymbol{u} \cdot \nabla \omega=v_{p} \nabla^{2 p} \omega-\alpha \omega+f
$$

in a square box of size $L=2 \pi$ with periodic boundary conditions using a fully dealiased pseudospectral code with second-order Runge-Kutta time stepping [34]. We also use a $p=8$ hyperviscous dissipation in order to extend the inertial range. The friction term proportional to $\alpha$ is necessary to avoid condensation of energy at the largest scale and to reach a statistically stationary state. The flow is generated by a small scale, $\delta$ correlated in time random forcing $f$, concentrated at the scale $\ell_{f}$, leading to an inverse energy cascade with flux $\varepsilon=2 \alpha u_{\mathrm{rms}}^{2}$. This defines the forcing time scale $\tau_{f}=\left(\ell_{f}^{2} / \varepsilon\right)^{1 / 3}$ which is used to rescale temporal variables.

For each value of $\tau_{p}$, we inject $M=65536$ particles with random initial positions $\boldsymbol{x}^{p}(0)$ and $\boldsymbol{v}^{p}(0)=\mathbf{0}$. Particles are advected according to

$$
\ddot{\boldsymbol{x}}^{p}(t) \equiv \dot{\boldsymbol{v}}^{p}(t)=\frac{1}{\tau_{p}}\left[\boldsymbol{u}\left(\boldsymbol{x}^{p}(t), t\right)-\boldsymbol{v}^{p}(t)\right]
$$

for a large-scale time until their distribution in the phase space become stationary, after which we collect their trajectories for a time $T=540 \tau_{f}$ [15]. The simulations were performed at a resolution of $1024^{2}$ with forcing centered on mode 320 . The inverse cascade inertial range extended to large scale $L=u_{\mathrm{rms}} /(2 \alpha)$, corresponding to a large-scale time $\tau_{L}=1 /(2 \alpha)$.

The statistics of particle separation, both forward and backward in time, are computed offline from these trajectories by looking, at each time, at particle pairs which are at the reference separation $\xi$. In what follows, the Stokes number is defined via the characteristic forcing time scale $S t=\tau_{p} / \tau_{f}$. 

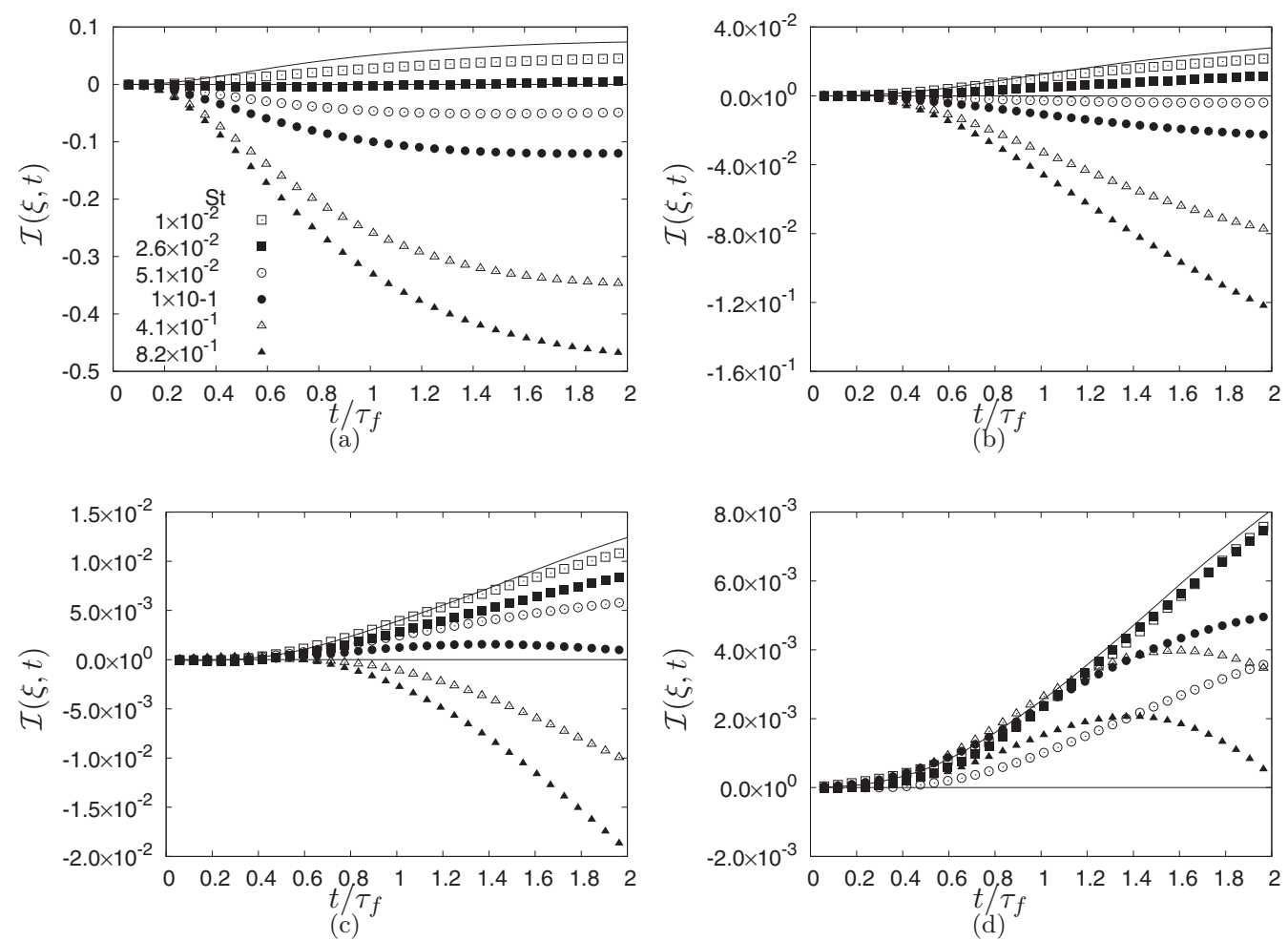

FIG. 1. DNS results for $\mathcal{I}(\xi, t)$ for various St and (a) $\xi / \ell_{f}=2$, (b) $\xi / \ell_{f}=5$, (c) $\xi / \ell_{f}=8$, and (d) $\xi / \ell_{f}=$ 10. Solid line denotes $\mathrm{St}=0$ results, and the horizontal line indicates $\mathcal{I}=0$.

In Fig. 1, we consider results for

$$
\mathcal{I}(\xi, t) \equiv \frac{\left\langle\left\|\boldsymbol{r}^{p}(t)\right\|^{2}-\left\|\boldsymbol{r}^{p}(-t)\right\|^{2}\right\rangle_{\xi}}{\left\langle\left\|\boldsymbol{r}^{p}(-t)\right\|^{2}\right\rangle_{\xi}}
$$

defined such that $\mathcal{I}(\xi, t)>0$ denotes FIT is faster than BIT dispersion, $\mathcal{I}(\xi, t)<0$ denotes BIT is faster than FIT dispersion, and $\mathcal{I}(\xi, t)=0$ denotes reversible dispersion (also, trivially, $\mathcal{I}(\xi, 0) \equiv 0$ ).

The results confirm the theoretical prediction of Sec. II, showing (for a given $\xi$ ) a transition from $\mathcal{I}<0$ to $\mathcal{I}>0$ as St is increased.

The results also show that for a given $\mathrm{St}$, the sign of $\mathcal{I}$ can change as $\xi$ is increased. This is because for fixed St, as $\xi$ is increased (and therefore $\mathrm{St}_{\xi}$ decreased), the NLIM weakens, and at sufficiently large scales, $\mathrm{St}_{\xi}$ becomes small enough for the LIM to dominate, giving $\mathcal{I}>0$.

It is interesting to note that the irreversibility inversions occur even when St is small. This is not in contradiction to the arguments in Sec. II since the regimes $\mathrm{St}_{\xi} \ll 1$ and $\mathrm{St}_{\xi} \geqslant O(1)$ are merely asymptotically defined, and the latter range is simply intended to denote the range over which the NLIM is expected to operate. We expect that the sign of $\mathcal{I}$ can change even for small $\mathrm{St}_{\xi}$ because the fluid energy flux in 2D turbulence is very small. As a result, only a small contribution from the path-history effect is needed to reverse the sign of the particle-pair energy flux, and hence reverse the sign of $\mathcal{I}$. This can also be understood in light of results in Ref. [11] that show that for 3D turbulence, the effect of the path-history mechanism on the odd-order moments of $\boldsymbol{w}^{p}(t)$ is very strong even for $\mathrm{St} \ll 1$, in which regime its effect on the even-order moments is small.

In Fig. 2(a), we plot $\left|\left\langle\left\|\boldsymbol{r}^{p}(t)\right\|^{2}-\left\|\boldsymbol{r}^{p}(-t)\right\|^{2}\right\rangle_{\xi}\right|$ in order to test the prediction of Sec. II that for small $t,\left\langle\left\|\boldsymbol{r}^{p}(t)\right\|^{2}-\left\|\boldsymbol{r}^{p}(-t)\right\|^{2}\right\rangle_{\xi} \propto t^{3}$. The results confirm the short-time $t^{3}$ growth of 

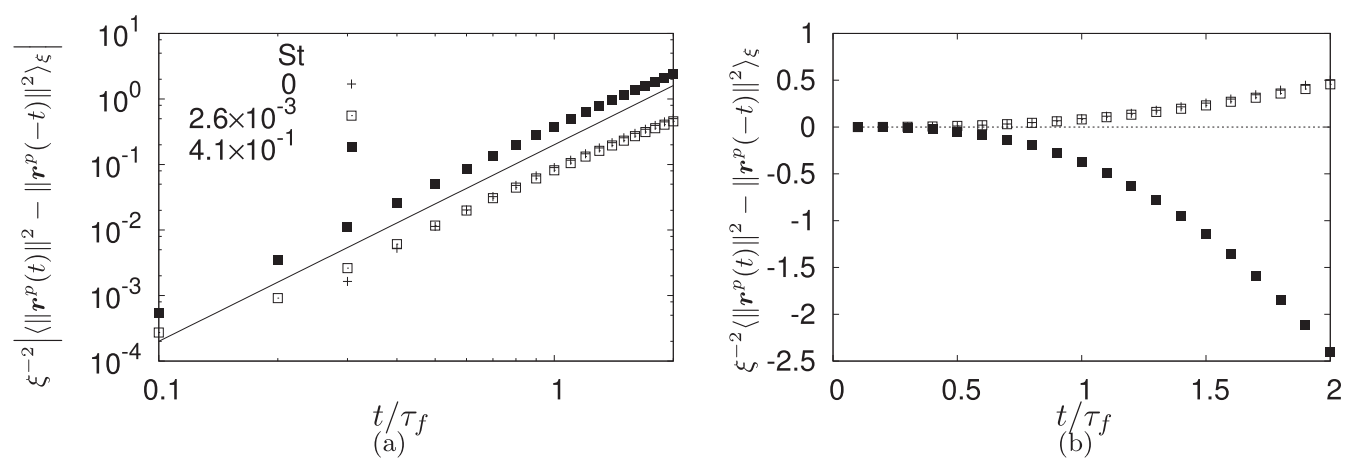

FIG. 2. DNS results for (a) $\xi^{-2}\left|\left\langle\left\|\boldsymbol{r}^{p}(t)\right\|^{2}-\left\|\boldsymbol{r}^{p}(-t)\right\|^{2}\right\rangle_{\xi}\right|$ and (b) $\xi^{-2}\left\langle\left\|\boldsymbol{r}^{p}(t)\right\|^{2}-\left\|\boldsymbol{r}^{p}(-t)\right\|^{2}\right\rangle_{\xi}$ for various $\mathrm{St}$ and $\xi / \ell_{f}=2$. Whereas plot (a) emphasizes the $t^{3}$ scaling, plot (b) reveals the change in sign of the short-time behavior of $\left\langle\left\|\boldsymbol{r}^{p}(t)\right\|^{2}-\left\|\boldsymbol{r}^{p}(-t)\right\|^{2}\right\rangle_{\xi}$ as $\mathrm{St}$ is increased, signifying the irreversibility inversion.

$\left\langle\left\|\boldsymbol{r}^{p}(t)\right\|^{2}-\left\|\boldsymbol{r}^{p}(-t)\right\|^{2}\right\rangle_{\xi}$ well, for St $\geqslant 0$. In Fig. 2(b), we highlight the change in sign of $\left\langle\left\|\boldsymbol{r}^{p}(t)\right\|^{2}-\left\|\boldsymbol{r}^{p}(-t)\right\|^{2}\right\rangle_{\xi}$ in the short-time $t^{3}$ regime, as St is increased.

We now use the DNS data to test our theoretical explanations for the dispersion irreversibility. First, we assumed that $\left\langle\left\|\boldsymbol{r}^{p}(t)\right\|^{2}\right\rangle_{\xi}$ is dominated by the behavior of particle pairs that are separating, and therefore have $w_{\|}^{p}>0$, whereas $\left\langle\left\|\boldsymbol{r}^{p}(-t)\right\|^{2}\right\rangle_{\xi}$ is dominated by the behavior of particle pairs that are approaching and therefore have $w_{\|}^{p}<0$ [2]. One way to test this assumption is to compute from the DNS the quantities

$$
\mathcal{J}(\xi, t) \equiv\left\langle\left[w_{\|}^{p}(t)\right]^{3}\right\rangle_{r^{p}(0)=\xi}, \quad \mathcal{J}(\xi,-t) \equiv\left\langle\left[w_{\|}^{p}(-t)\right]^{3}\right\rangle_{r^{p}(0)=\xi}
$$

The results in Fig. 3 clearly validate our argument, showing $\mathcal{J}(\xi, t)>0$ and $\mathcal{J}(\xi,-t)<0$.

Second, we argued that at any given separation $r$, particle pairs that are moving together should do so with relative velocities whose magnitudes are characteristically different from those of particles that are moving apart. In other words, the PDF of $w_{\|}^{p}$ should be skewed, both because of the presence of dynamical fluxes in the turbulent velocity field, and because of the path-history effect. Our arguments predict that in $2 \mathrm{D}$ turbulence, when $\mathrm{St}_{\xi} \ll 1$, the skewness should be positive, but when $\mathrm{St}_{\xi}$ is large enough for the nonlocal inertial particle dynamics to dominate the flux, the skewness should become negative. The quantity $\mathcal{J}$ is not appropriate for testing this part of the argument; since the particles disperse at different rates FIT and BIT, then for a given $t, \mathcal{J}(\xi, t)$ and $\mathcal{J}(\xi,-t)$ will be associated with the particle relative velocities at different separations. Instead, the appropriate quantity to test
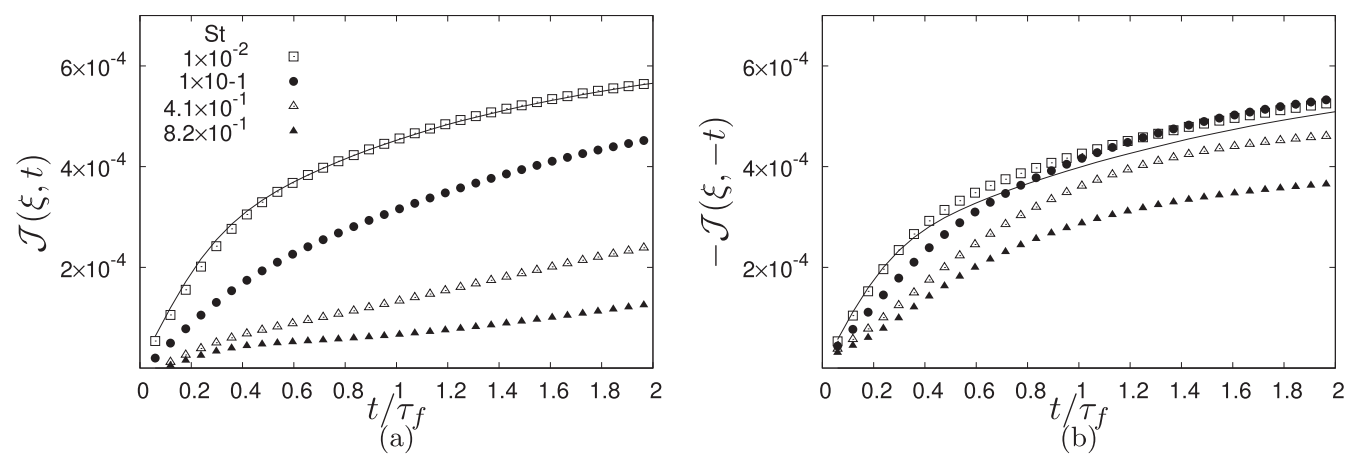

FIG. 3. DNS results for (a) $\mathcal{J}(\xi, t)$ and (b) $\mathcal{J}(\xi,-t)$ for various St and $\xi / \ell_{f}=2$. 


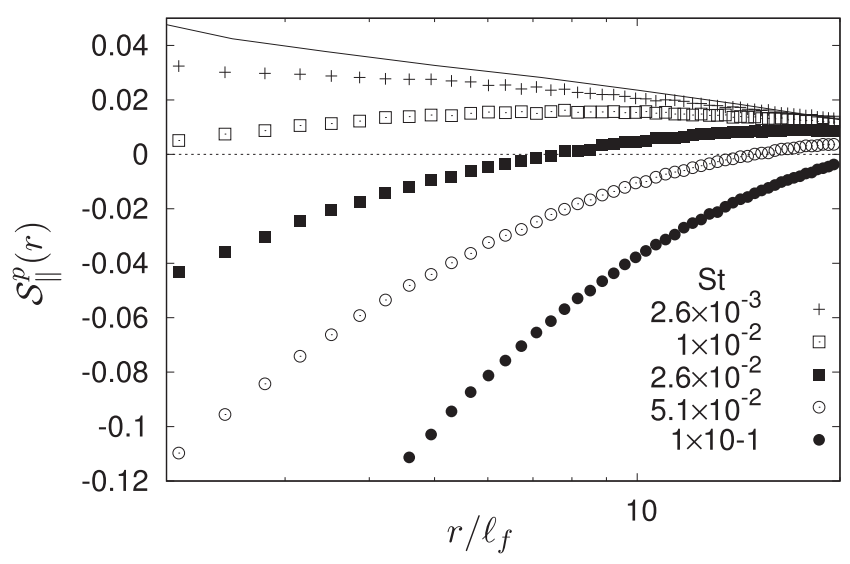

FIG. 4. DNS results for $\mathcal{S}_{\|}^{p}(r)$ for various $\mathrm{St}$. The continuous line is the skewness of the fluid velocity (i.e., $\mathrm{St}=0)$, given for reference. Note the change in sign of $\mathcal{S}_{\|}^{p}(r)$ as St is increased.

this argument is

$$
\mathcal{S}_{\|}^{p}(r) \equiv\left\langle\left[w_{\|}^{p}(t)\right]^{3}\right\rangle_{r} g /\left\langle\left[w_{\|}^{p}(t)\right]^{2}\right\rangle_{r}^{3 / 2},
$$

i.e., the skewness of the PDF of $w_{\|}^{p}$ at fixed separation $r$.

The results in Fig. 4 confirm the predictions; for fluid particles $\mathcal{S}_{\|}^{p}>0 \forall r$, whereas $\mathcal{S}_{\|}^{p}$ can be both positive or negative for inertial particles, depending upon $r$ and St. Also in agreement with the theoretical predictions, for a given $\mathrm{St}, \mathcal{S}_{\|}^{p}$ can change sign as $r$ is varied. This is due to the variation in $\mathrm{St}_{r}$ with $r$ : At small enough $r, \mathrm{St}_{r}$ can be large enough for the nonlocal inertial effects to dominate, yielding $\mathcal{S}_{\|}^{p}<0$. However, as $r$ is increased, $\mathrm{St}_{r}$ decreases, and when $\mathrm{St}_{r}$ becomes sufficiently small the local turbulence dominates the inertial particle behavior, and $\mathcal{S}_{\|}^{p}>0$.

The results in Fig. 4 are of significant interest even beyond the problem of dispersion irreversibility. In particular, they show that even though the fluid exhibits an inverse energy flux in 2D, particles transported by such a flow may in fact exhibit a downscale/direct energy flux, depending on their inertia. This nontrivial behavior is yet another manifestation of the complexity and subtlety of inertial particle dynamics in turbulent flows.

It is important to emphasize that the qualitative explanations given in Sec. II connect the irreversibility of the dispersion to the asymmetry of the PDF of $w_{\|}^{p}$ for any time in the dispersion process. However, at present we are only able to demonstrate this analytically in the limit $t \rightarrow 0$, through the analysis in Sec. II. An important point for future work is to demonstrate this dependence analytically for arbitrary $t$, which is a very challenging task.

We close this section with a comment on the relationship between the local irreversibility mechanism and the dynamical cascade processes in operation in $2 \mathrm{D}$ turbulence. According to the theoretical explanations in Ref. [2] and those in Sec. II, the local irreversibility mechanism is connected to the sign of the fluid energy flux, irrespective of the underlying dynamics responsible for this. For example, in the present case of 2D turbulence, our explanations predict that FIT is faster than BIT dispersion for $\mathrm{St}=0$, irrespective of whether the particle separation lies in the regime of the inverse energy cascade or the direct enstrophy cascade. All that matters for this prediction is that the flux is positive. Our explanation is therefore somewhat different than the explanation proposed in Ref. [13], which connected the irreversibility of fluid particle-pair dispersion in 2D turbulence with the dynamics of the inverse energy cascade itself. However, we emphasize that through the local irreversibility mechanism, fluid particle-pair dispersion would be irreversible even in kinematically constructed flow fields, provided only that the PDF of $\Delta u_{\|}$is asymmetric and that the Lagrangian time scales of the flow are finite. 


\section{CONCLUSIONS}

In this paper, we have supplemented a qualitative argument presented in a recent paper with a new quantitative prediction for the irreversibility of inertial particle dispersion in $2 \mathrm{D}$ turbulence. Using DNS data we have confirmed the predictions that in 2D turbulence, the forward dispersion of particle pairs is faster than the backward dispersion, until the particle inertia passes a certain threshold, and then the backward dispersion becomes faster than the forward dispersion. The confirmation of the prediction lends strong support to our arguments that the irreversibility of inertial particle dispersion in turbulence is governed by two completely distinct physical mechanisms, whose relative influence depends upon the inertia of the particles. More generally, the results are also of interest since they reveal that in turbulence flows with an inverse energy cascade, inertial particles may exhibit a downscale flux of kinetic energy because of their nonlocal-in-time dynamics. These results could therefore be significant in understanding and modeling the motion of inertial particles in certain geophysical and astrophysical flows that exhibit quasi-2D dynamics.

Among the simplifying assumptions made in prescribing (1) as the particle equation of motion, the neglect of the Basset history force [22] deserves particular attention, since this term has been found to produce nontrivial effects on particle clustering and acceleration statistics $[35,36]$, at least some of which are still not completely understood. A quantitative investigation of the irreversible dispersion of particles governed by more complete models for their equation of motion is left to future investigations.

[1] G. Falkovich, K. Gawedzki, and M. Vergassola, Particles and fields in fluid turbulence, Rev. Mod. Phys. 73, 913 (2001).

[2] A. D. Bragg, P. J. Ireland, and L. R. Collins, Forward and backward in time dispersion of fluid and inertial particles in isotropic turbulence, Phys. Fluids 28, 013305 (2016).

[3] G. Falkovich and A. Frishman, Single Flow Snapshot Reveals the Future and the Past of Pairs of Particles in Turbulence, Phys. Rev. Lett. 110, 214502 (2013).

[4] J. Jucha, H. Xu, A. Pumir, and E. Bodenschatz, Time-Reversal-Symmetry Breaking in Turbulence, Phys. Rev. Lett. 113, 054501 (2014).

[5] H. Xu, A. Pumir, G. Falkovich, E. Bodenschatz, M. Shats, H. Xia, N. Francois, and G. Boffetta, Flight-crash events in turbulence, Proc. Natl. Acad. Sci. USA 111, 7558 (2014).

[6] A. Frishman, G. Boffetta, F. De Lillo, and A. Liberzon, Statistical conservation law in two- and threedimensional turbulent flows, Phys. Rev. E 91, 033018 (2015).

[7] H. Xu, A. Pumir, and E. Bodenschatz, Lagrangian view of time irreversibility of fluid turbulence, Sci. Chi. Phys. Mech. 59, 1 (2015).

[8] D. Buaria, B. L. Sawford, and P. K. Yeung, Characteristics of backward and forward two-particle relative dispersion in turbulence at different reynolds numbers, Phys. Fluids 27, 105101 (2015).

[9] D. Buaria, P. K. Yeung, and B. L. Sawford, A Lagrangian study of turbulent mixing: Forward and backward dispersion of molecular trajectories in isotropic turbulence, J. Fluid Mech. 799, 352 (2016).

[10] A. Pumir, H. Xu, E. Bodenschatz, and R. Grauer, Single-Particle Motion and Vortex Stretching in ThreeDimensional Turbulent Flows, Phys. Rev. Lett. 116, 124502 (2016).

[11] A. D. Bragg, Analysis of the forward and backward in time pair-separation probability density functions for inertial particles in isotropic turbulence, J. Fluid Mech. 830, 63 (2017).

[12] L. Biferale, A. S. Lanotte, R. Scatamacchia, and F. Toschi, Intermittency in the relative separations of tracers and of heavy particles in turbulent flows, J. Fluid Mech. 757, 550 (2014).

[13] T. Faber and J. C. Vassilicos, Turbulent pair separation due to multiscale stagnation point structure and its time asymmetry in two-dimensional turbulence, Phys. Fluids 21, 015106 (2009).

[14] G. Boffetta and R. E. Ecke, Two-dimensional turbulence, Annu. Rev. Fluid Mech. 44, 427 (2012). 
[15] G. Boffetta, F. De Lillo, and A. Gamba, Large scale inhomogeneity of inertial particles in turbulent flows, Phys. Fluids 16, L20 (2004).

[16] L. Chen, S. Gogo, and J. C. Vassilicos, Turbulent clustering of stagnation points and inertial particles, J. Fluid Mech. 553, 143 (2006).

[17] S. Goto and J. C. Vassilicos, Self-similar clustering of inertial particles and zero-acceleration points in fully developed two-dimensional turbulence, Phys. Fluids 18, 115103 (2006).

[18] S. Goto and J. C. Vassilicos, Sweep-Stick Mechanism of Heavy Particle Clustering in Fluid Turbulence, Phys. Rev. Lett. 100, 054503 (2008).

[19] S. W. Coleman and J. C. Vassilicos, A unified sweep-stick mechanism to explain particle clustering in twoand three-dimensional homogeneous, isotropic turbulence, Phys. Fluids 21, 113301 (2009).

[20] S. Musacchio and G. Boffetta, Split energy cascade in turbulent thin fluid layers, Phys. Fluids 29, 111106 (2017).

[21] A. Bracco, P. H. Chavanis, A. Provenzale, and E. A. Spiegel, Particle aggregation in a turbulent Keplerian flow, Phys. Fluids 11, 2280 (1999).

[22] M. R. Maxey and J. J. Riley, Equation of motion for a small rigid sphere in a nonuniform flow, Phys. Fluids 26, 883 (1983).

[23] This path-history effect arises from the Stokes drag force and is not related to the Basset history force that appears in the more general particle equation of motion [22].

[24] A. D. Bragg and L. R. Collins, New insights from comparing statistical theories for inertial particles in turbulence: I. Spatial distribution of particles, New J. Phys. 16, 055013 (2014).

[25] A. D. Bragg and L. R. Collins, New insights from comparing statistical theories for inertial particles in turbulence: II. Relative velocities, New J. Phys. 16, 055014 (2014).

[26] E. Lindborg, Can the atmospheric kinetic energy spectrum be explained by two-dimensional turbulence? J. Fluid Mech. 388, 259 (1999).

[27] A. D. Bragg, P. J. Ireland, and L. R. Collins, Mechanisms for the clustering of inertial particles in the inertial range of isotropic turbulence, Phys. Rev. E 92, 023029 (2015).

[28] P. J. Ireland, A. D. Bragg, and L. R. Collins, The effect of Reynolds number on inertial particle dynamics in isotropic turbulence. Part 1. Simulations without gravitational effects, J. Fluid Mech. 796, 617 (2016).

[29] M. Wilkinson and B. Mehlig, Caustics in turbulent aerosols, Europhys. Lett. 71, 186 (2005).

[30] M. R. Maxey, The gravitational settling of aerosol particles in homogeneous turbulence and random flow fields, J. Fluid Mech. 174, 441 (1987).

[31] A. D. Bragg, P. J. Ireland, and L. R. Collins, On the relationship between the non-local clustering mechanism and preferential concentration, J. Fluid Mech. 780, 327 (2015).

[32] K. Gustavsson and B. Mehlig, Ergodic and non-ergodic clustering of inertial particles, Europhys. Lett. 96, 60012 (2011).

[33] K. Gustavsson and B. Mehlig, Distribution of relative velocities in turbulent aerosols, Phys. Rev. E 84, 045304(R) (2011).

[34] G. Boffetta, A. Celani, and M. Vergassola, Inverse energy cascade in two-dimensional turbulence: Deviations from Gaussian behavior, Phys. Rev. E 61, R29(R) (2000).

[35] S. Olivieri, F. Picano, G. Sardina, D. Iudicone, and L. Brandt, The effect of the Basset history force on particle clustering in homogeneous and isotropic turbulence, Phys. Fluids 26, 041704 (2014).

[36] A. Daitche, On the role of the history force for inertial particles in turbulence, J. Fluid Mech. 782, 567 (2015). 